to date in many diseases, and also metabolic, hormonal, and other physiologic effects. The Manual runs to close on 1,600 pages, but is nevertheless within the limits of size and weight which justify its description as a handbook, owing to the thinness of the paper and the general arrangement. It will be found of the greatest assistance to the busy general practitioner to whom it may be strongly recommended.

C. W. BuCKLEY.

\title{
HOSPITAL REPORTS
}

Reports have been received of the work which is being done in some of the hospitals specializing in the treatment of rheumatic diseases, and in research, clinical and laboratory. These furnish most encouraging evidence of the progress which is being made in some centres in the campaign against rheumatism.

West London Hospital: Department of Rheumatic Diseases. Annual Report for 1949.

Great progress is being made at this centre, which possesses a large staff of skilled workers including holders of Research Fellowships and Studentships. A peripheral clinic established in 1946 is doing good work, and co-operation has been established with the British Post-graduate Hospital. Twelve beds are available for in-patients at the West London Hospital and the Hospital of St. John and St. Elizabeth, St. John's Wood, and further beds are shortly to be provided at St. Mary Abbots Hospital, Kensington.

A special research department has been established to which the Medical Research Council has made a large grant to aid study of the effects of cortisone in rheumatic disease and generous grants have also been made by the Dan Mason Research Fellowship of the West London Hospital Medical School; in addition Roche Products Limited have, through the Empire Rheumatism Council, presented a Roche Fellowship tenable for two years for a steroid chemist. The Report contains interesting and important papers by members of the staff, notably one by Dr. P. M. F. Bishop on the bio-chemical and endocrine aspects of cortisone, and one by Dr. A. A. Henly on the adrenocorticotrophic hormone. A list of papers from members of the staff, published elsewhere, is also included. A grant from the British Schering Co. Ltd., has rendered possible this very full and well-produced report.

The Royal Free Hospital: Unit of Rheumatology. Report for 1949.

Important work is being done at this centre; statistics are given of the numbers treated and the results, and the figures show very encouraging improvement in the results of treatment of ankylosing spondylitis. It is pointed out that the long waiting list militates against efficient treatment in hospital of rheumatoid arthritis, cases lose ground often to a serious degree while waiting for admission and the shortage of beds for chronic cases is also a serious matter. Valuable work is being done by the Almoners and useful statistics have been compiled showing that only a small proportion of the cases which have been under treatment relapse. Special attention is being given to the treatment of certain deformities and to rehabilitation, and research in various directions is being actively carried out.

Royal National Hospital for Rheumatic Diseases, Bath. Vol. 3, 1948-49.

Here the formation of a research unit in connection with the South-Western and Oxford Regional Hospital Boards has been an important development; the unit has fifty beds and its own laboratory, and a further seventy beds are available for teaching and research. The staff of the research unit consists of four Research Fellows, and the junior hospital staff has been expanded to include a Senior Registrar, two Registrars, and three House Physicians. Further developments, especially in the Manor Hospital for orthopaedic work, are likely in the near future. The Report also includes reprints of papers contributed by members of the staff to various journals.

C.W.B. 